\title{
Study on the Micro-course Mobile Teaching System Based on Android Platform
}

\author{
Dingyu Song
}

Department of educational affairs of Nanyang Institute of Technology, Nanyang, Henan, 473000

Keywords: Micro-course; Android platform; mobile teaching system

\begin{abstract}
Mobile learning has increasingly become a popular new learning model, and APP applications have gradually become a learning tool. The high market share of Android system makes it easier to promote Android-based applications. Because of its small and clear learning goals, micro lessons can improve the efficiency of learners' learning and quickly become the focus of attention in the current education community. The paper expounds the research background, analyzes the system function, introduces the related technology, discusses the online video playback and reading data modules of the system client and the implementation details of the server-side learning data module. The entire system runs well and has a good guiding significance for practical applications.
\end{abstract}

\section{Introduction}

The mobile learning platform must be able to allow learners to learn anytime, anywhere, and provide support for communication between teachers and students. With the development of wireless networks, "mobile learning" based on mobile Internet technology has increasingly become a new learning model that is considered and pursued by learners. Most of the existing micro-courses are based on traditional website development technologies. Micro-courses based on mobile Internet technologies are in a stage of gradual development and improvement. Building mobile micro-courses through mobile internet technology is bound to become a trend and research hotspot. The mobile phone operating system is the most widely used non-Android. In the fourth quarter of 2013, Android-based terminals accounted for $78.1 \%$ of the global market share. Android is deeply concerned by young people because of its advantages of convenience, openness, and low cost. Android application promotion is easier, which is conducive to mobile learning.

\section{System and Its Function Analysis}

Using the mobile micro-course platform client, users can select knowledge points and view their micro-videos and other reference documents online. The system needs to provide online communication functions such as commentary videos. Submitting comments first requires identity confirmation. The server of the mobile micro-learning platform can manage micro-course resources, such as the management of curriculum knowledge points, micro-video corresponding to knowledge points, and reference document management. Android-based mobile micro-course platform is divided into two parts: client system and server system. Platform client to achieve the following basic functions: User management. It involves user registration, login, password modification and retrieval. User registration: Some functions require the user to log in and authenticate only after the user has logged in. Therefore, the system must implement the new user registration function. After the user logs in, the user name and password are correct. You can enter the system, remember the password, and automatically log in. Password recovery: when the user password after forgetting, you can retrieve the password in the form of a text message. Password modification: For security reasons, users can modify their own passwords. System settings. Including software version detection and upgrade, feedback and other functions. Course study. Including video playback, reference document reading and comment interaction. Video playback: After the user selects the knowledge point, the user can view the corresponding micro video online; refer to the document reading: the user can read the corresponding reference document after selecting the knowledge 
point; the comment interaction: the student can comment on the micro video corresponding to each knowledge point. So as to achieve the effect of teacher-student interaction. The mobile micro-services platform server must implement the following basic functions: Web Service interface. The Web Service interface is a bridge connecting the Android client and the server backend database. The Web Service interface is responsible for the interaction between the backend database and the foreground client program. According to the analysis, the Web Service interface in this system needs to provide basic functions such as reading all knowledge points of a course, obtaining the corresponding comment information of a certain knowledge point, user login verification, and adding video comment information. Micro-curriculum resource management. For the client to use the video, documents and other data needs to be stored in the back-end database, taking into account the use of computer operation is more convenient, it is necessary to develop the corresponding resource management system, including the knowledge of the course management, knowledge of the corresponding micro-video and reference documents Management and so on.

Web Service is an interoperable, self-describing, loosely-coupled, and programmable Web application that supports different platforms under network conditions. It uses HTTP to process requests and triggers calls. Web Service is the external interface of the system. Different applications on different platforms can use this technology to complete the data exchange and integration without the intervention of third-party software and hardware. With Web Services, we can call methods on a remote server just like calling a native method. We don't need to care about what language the remote method is written in, nor what platform the remote method is based on. JSON technology JavaScript Object Notation, referred to as JSON, means JavaScript (JS) object representation, which is a lightweight data exchange format. JSON is a special JS, plain text format, completely separate from the programming language. The reason why JSON is a great data exchange language is not unrelated to these features. It is not only easier to read, but also easier for machines to parse. JSON is more extensible than the XML language. Because JSON has an absolute advantage in storing JS composite objects, JSON has the advantage that XML cannot replace it. The effective data rate is another difference between JSON and XML. The JSON structure is clearer and there is less duplicate information. It is not necessary to have a strict closed tag. Therefore, it is more efficient to transmit JSON data packets over the network and reduce the pressure on network transmission. Handler message mechanism If the program does not respond for more than 5 seconds, Android will prompt the user to close the application. The time-consuming operation should be placed in the child thread. After the child thread is processed, the interface in the main thread is updated. . It should be noted that the controls in the Activity are not thread-safe, and control-related operations are forced on the main thread. Therefore, newly started threads cannot operate on them, that is, child threads cannot directly access the main thread's interface controls. The Handler message mechanism can solve this problem. In simple terms, Handler is a processing object used to exchange data between processes. Handler is like a link. Any process that can get a Handler of a process can call its sendMessage method to pass data to the process. So we can create a thread with a Handler in the main thread when dealing with multi-threading problems. When the thread finishes those time-consuming operations, it uses the Handler to send data to the main thread. After the main thread receives the message, it will perform updates, for example. Corresponding processing such as interface.

\section{System Design}

Information Management: Designed specifically for administrators, it is to realize the initialization of data in the system and obtain the database. Such as the basic information management of teachers and students, platform settings and platform security management. Resource Push: The curriculum resources in the system are standardized by teachers, which facilitates students' self-study after class, teachers build courses and upload corresponding micro-curriculum resources. Further education: Provides teachers with micro-course video and resource additions, deletions, and student online questioning platforms. Resource review: The teacher reviews students' shared micro-curriculum resources, such as whether the size, theme, 
content, and format of the uploaded resources meet the requirements of the system's shared resources.

Course Study: Micro-curriculum learning resources mainly include related topics such as micro-course video, online test bank, student discussion area, etc. Micro-course video is the most important part of the curriculum resources. When a video is opened for learning, download. The video is cached locally and the video player of the Android system is called to play the video. The teacher-student exchange mainly interacts through the discussion area, mainly to solve student learning problems. Online testing: For the online testing of a course, it is mainly about the resource of the question bank of a certain course. Students can conduct self-testing, and can evaluate the question bank resources and discuss and communicate with other students. Resource Push: The curriculum resources in the system are standardized by teachers, which facilitates students' self-study after class, teachers build courses and upload corresponding micro-curriculum resources.

This software development is based on the Android system environment, using the development technology based on the JAVA platform, first need to install the JAVA development tool JDK. The server program uses Eclipse+Tomcat and the system database uses SQL Server 2008. The client program mainly uses Eclipse+ADT+SDK. The Android SDK provided by the SDK SDK, which contains APIs for developing Android applications. This toolkit is required when developing Android-based software. Eclipse development of Java application software, one of the IDE tools, features open source, super plug-in features, compatible with multiple language development. ADTADT full name is "Android Development Tools", is an Eclipse plug-in provided by Google, Used to provide a powerful, integrated An-droid development environment in Eclipse.

SQL Server 2008 was released on Microsoft's data platform, which provides a highly trusted, efficient, intelligent data platform. SQL Server 2008 provides a rich set of integration services that can perform operations such as querying, searching, synchronizing, reporting, and analyzing data. SQL Server 2008 allows the use of data in custom applications developed by Microsoft.NET and Vi-sual Studio to use data in service-oriented architecture (SOA) and business processes conducted through Microsoft BizTalk Server.

The micro-course sharing platform consists of an Android mobile terminal, a SQL server 2008 database, and a background server. Android mobile terminal as the front desk of the platform, page design, code programming in eclipse using the Java language; SQL server 2008 database is mainly used to store the data displayed on the client, each time the client's access is through the mobile network Platform and back-end servers perform data updates. The service area is mainly responsible for storing user information, micro class resource push information, and so on. The Android client interacts with the server to perform json data exchange, mainly by writing and parsing json data. The Android client interacts with the server to perform json data exchanges. If the server writes and parses the data of the son situation, Server creates a new ServerSock event, uses the accept function until it connects to the client, and the client creates a new socket. And request to connect with the server side; The server side to take over the client's requirements, create a new socket and the client connection; On a separate thread, the establishment of the link socket swap; The server continues to wait for the next link.

\section{Conclusion}

This article first describes the design and implementation of a micro-course sharing platform based on Android, and then elaborates the functions of the main modules of the APP, the data exchange methods of the client and server, the features and technical implementation points of the background management module, and profoundly understands "Micro-curriculum" has become a hot spot in the current new curriculum reform. Therefore, micro-classes and mobile platforms are organically integrated so that users can learn in fragmented time, such as lining up between classes, buses, and even dining in the cafeteria. The network can also be used for learning in a short period of time, which can improve the user's learning efficiency. 


\section{References}

[1] Shieh, D. These Lectures Are Gone in 60 Seconds [J]. Chronicleof Higher Education, 2009(3): A1, A13.

[2] Liang Leming, Cao Qiaoqiao, Zhang Baohui. Research on Micro-curriculum Design Patterns: A Comparative Analysis of Micro-curriculum at Home and Abroad [J]. Open Education Research, 2013(01): 65-66.

[3] Hu Tiesheng. "Micro-curriculum": New Trends in the Development of Regional Education Information Resources [J]. Electrification Education Research, 2011(10):61-65.

[4] Guo Zhongnan. Application of Web Service in Android-based Mobile Educational Administration System [J]. Fujian Computer, 2015(11):133-134.

[5] Yang Jie. Multithreading Technology Based on Android [J]. Computer Knowledge and Technology, 2013(18):4251-4254. 